\title{
LOS CUMPLIDOS EN MEdellín y SU ÁREA METROPOLITANA (El Valle de Aburrá)*
}

\author{
Sergio Alonso Lopera Medina*
}

\begin{abstract}
Resumen
Este artículo reporta el análisis de uso de los cumplidos en el área metropolitana de Medellín-Colombia. Como técnica de investigación se combinaron el método de campo y el método de laboratorio (Jucker, 2009), y se analizaron 2 fuentes de información: cuestionario de completación (DCT) y diario de campo. Los resultados indican que los cumplidos de apariencia son los más comunes y la forma de realización es explícita. Además, el uso de interjecciones, los adverbios de intensificación tan y muy y el dialecto social Parlache son frecuentes en los cumplidos.
\end{abstract}

Palabras clave: cumplidos, realización explícita, Medellín.

\section{Compliments in Medellín and its metropolitan area (El Valle del Aburrá)}

\begin{abstract}
This article reports the analysis of compliment use in the metropolitan area of MedellínColombia. Both the field method and the laboratory method (Jucker, 2009) were combined as research technique. Two sources of information were analyzed: a field diary and a Discourse Completion Test (DCT). Results support that compliments of appearance are the most common ones. Also, the most common way of compliment production is explicit. Finally, the use of interjections, the adverbs of intensification tan y muy (so and very), and the social dialect Parlache are frequent in the compliments.
\end{abstract}

Key words: compliments, explicit production, Medellín.

Recibido: 04-01-2013 Aceptado: 27-06-2013

* Este articulo hace parte de la investigación "análisis sociolingüístico y pragmático de los cumplidos entre la población universitaria en Medellín, Colombia". La investigación es apoyada por el Programa de Estrategia de Sostenibilidad de Grupos de investigación de la Universidad de Antioquia 2013-2014.

** Miembro del grupo de investigación GELIR, Facultad de Comunicaciones. Estudiante de Doctorado en Lingüística, profesor tiempo completo Escuela de Idiomas, Universidad de Antioquia UdeA, Medellín, Colombia. slopera@idiomas.udea.edu.co 


\section{Introducción}

Dentro de los actos expresivos, los cumplidos son utilizados en las culturas como una manera de elogiar (Bustos, 2007). Cuando se comunica un cumplido, ciertas normas pragmáticas se ponen en escena, siendo la competencia lingüística un eje principal para la ejecución o recepción del cumplido. Los cumplidos se pueden mirar como una manera de establecer un buen ambiente de interacción entre los participantes.

Este artículo analiza algunas muestras de los cumplidos en un cuestionario de completación (Discourse Completion Test-DCT) y en un diario de campo. Para el análisis se tuvieron en cuenta la categorización a nivel de objeto y forma de realización de los cumplidos (Bustos, 2007; Campo \& Zuluaga, 2000; Jucker, 2009; Rees-Miller, 2011; Manes \& Wolfson, 1981).

Este artículo se presenta de la siguiente manera: inicia con una revisión de la literatura; luego se contextualiza la metodología. Como tercer elemento se brinda el análisis con algunos comentarios y observaciones. Finalmente, se exponen las conclusiones y limitaciones del estudio.

\section{Revisión de la literatura}

La interacción lingüística se puede brindar en contextos formales e informales. En el primer contexto, se podría imaginar lugares donde prima la academia (universidades, auditorios, bibliotecas); en el segundo, se podría tener lugares tales como una cafetería, una tienda o un cine. En ambas situaciones se intercambia información lingüística donde los participantes tienden a seguir algunas normas sociales para evitar la agresividad entre ellos (Escandell, 1993).

Sin importar el contexto, los participantes producen unos actos de habla que en determinadas situaciones invitan a solicitar información, realizar una invitación, ofrecer una disculpa o hacer un cumplido. Estos a su vez involucran tres tipos de subactos: locucionario, ilocucionario y perlocucionario. Austin, (1962) y Searle (1969) incluyen los expresivos dentro de los subactos de habla ilocutivos y los define como elementos que expresan sentimientos y emociones: felicitar, agradecer, brindar un cumplido, entre otros. El usuario utiliza estos actos expresivos para realzar la imagen del interlocutor y, de esta manera, muestra interés y simpatía hacia el otro. 
Los cumplidos se utilizan usualmente como actos expresivos que se extienden en diferentes culturas y han sido estudiados por investigadores en diferentes contextos y momentos. Desde la década de los 70`s (Herbert, 1989; Holmes, 1986) han publicado varios estudios relacionados con el análisis sistemático de los cumplidos o respuestas a los cumplidos. Los campos de la lingüística como la pragmática, análisis del discurso y la sociolingüística han realizado estudios de los cumplidos teniendo como eje principal el análisis de los aspectos de fórmulas de cortesía que son usados por diferentes comunidades en sus contextos sociales.

La mayoría de los estudios sobre los cumplidos han sido desarrollados básicamente en América, Europa y Asia y en países de habla inglesa (Coulmas, 1980; Farghal \& Haggan 2006; Golato 2002; Herbert, 1986, 1987, 1989; Holmes, 1986, 1988; Holmes \& Brown, 1987; Lorenzo-Dus, 2001; Manes, 1983; Mustapha 2004; Norrick, 1980; Tang \& Zhang, 2009; Wolfson, 1983).

Los estudios del acto de habla de los cumplidos son escasos en el idioma español. Estudios en Costa Rica (Murillo, 2003), en Lima y Cuzco, Perú (De los Heros, 2001) sólo dan cuentan de un análisis sistemático donde el primero arroja como resultado que las personas usan los cumplidos con formas exclamativas y los segundos enfatizan el género y el poder relativo. Campo y Zuluaga $(2000,31)$ argumentan que los cumplidos en español se categorizan en habilidad y en apariencia. Las investigadoras documentaron que los hablantes del español hacen uso de los "piropos" como una forma de hacer un cumplido de apariencia en Colombia. Finalmente, Bustos (2007) sostiene que los hablantes españoles no utilizan con mayor frecuencia los cumplidos por temor a que se confundan con el piropo.

\section{Los cumplidos}

Los cumplidos son expresiones verbales que se utilizan no sólo para demostrar el comportamiento cortés, sino también para asegurar que los participantes están conscientes de que se valoran las opiniones dentro de un grupo sociocultural, conllevando a una solidaridad conjunta. Holmes $(1988,446)$ define a los cumplidos como "un acto de habla que implícita o explícitamente honra a otra persona diferente al hablante, usualmente al interlocutor, por su posesión, característica, habilidad, etc., y que es valorado positivamente tanto por el hablante como por el oyente" 
(traducción propia). Herbert (1997) argumenta que los cumplidos poseen dos condiciones principales: a) contienen una expresión de admiración por parte del interlocutor, b) involucran una posesión, una cualidad personal o un logro.

Jucker (2009) argumenta que los cumplidos están inmersos a nivel cultural y social y es por esto que, en ocasiones, unos cumplidos que son usados apropiadamente en una comunidad cultural específica pueden no serlo en comparación con otra.

\section{Categorización de los cumplidos}

Los investigadores clasifican o agrupan los cumplidos de diferentes maneras. La siguiente agrupación abarca una clasificación general de cumplidos planteada por diferentes investigadores (Bustos, 2007; Campo y Zuluaga, 2000; Jucker, 2009; Rees-Miller, 2011; Manes y Wolfson, 1981).

\section{Objeto del cumplido}

Apariencia: el hablante brinda una valoración positiva del físico de su interlocutor: ¡Cómo quedaste de bien así motilado'!

Posesión: el hablante brinda una valoración positiva a algún elemento poseído por su interlocutor: ;Ah, mija² qué bolso tan bonito!

Habilidad: el hablante brinda una valoración positiva de destreza: Huy

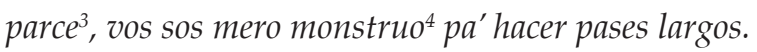

Personalidad: el hablante brinda una valoración de cualidad de personalidad: eres muy maduro para tu edad ${ }^{5}$.

1 Ejemplos tomados del corpus.

2 Mija es un vocativo.

3 Parce es supresión de parcero y se utiliza como vocativo. Parce hace parte del parlache y se define como "un dialecto social que nace y se despliega en los barrios populares de la ciudad de Medellín y en el área metropolitana del Valle de Aburrá, como una de las respuestas que los grupos sociales excluidos de la educación, la actividad laboral y la cultura dan a los otros sectores de la población, frente a los cuales se sienten fuertemente marginados" (Henao y Castañeda, 2001, p. 1-2). Las transformaciones sociales, culturales y laborales hicieron un cambio marcado de sectores poblacionales de Medellín y esto conllevó también a un cambio lingüístico en la ciudad. La fuerza de este dialecto trascendió las fronteras de los barrios populares y hoy aparecen expresiones en los diarios o programas humorísticos. El parlache se caracteriza por la transformación de palabras existentes en el idioma y crea unas nuevas. Por ejemplo hay adición de fonemas bus (buséfalo), supresión de fonemas compañero (ñero). También utiliza la inversión silábica de palabras frío (ofri), préstamo de términos de otras lenguas lo siento (ansorris), elementos onomatopéyicos campanero (tilín tilín), uso de prefijos mujer bonita (recuca), nombres de personas por semejanza fonética como robo (Roberto), metáfora terminar un trabajo (coronar), metonimia policia (verde).

4 Monstruo es un vocativo que indica grandeza.

5 Ejemplo inventado. 


\section{La forma de realización}

Jucker $(2009,1612)$ argumenta que el cumplido se puede hacer de una manera explícita, implícita o indirecta:

Explícita es cuando el hablante realiza un cumplido directamente: Entonces qué bizcocho ${ }^{6}$, cómo has estado, por qué tan perdido.

Implícita es cuando se infiere el cumplido aun si no se ha dicho explícitamente: jeh, quisiera tener un celular como el tuyo!

Indirecta es cuando el hablante se refiere a una tercera persona mencionando un cumplido: "Hey, mera moto la que compró el parcero de tu hermano".

\section{Metodología}

Los métodos lingüísticos de investigación enfatizados en la pragmática han sido basados en la intuición, experimentación y la observación. Clark y Bangerter $(2004,25$, citados en Jucker 2009, 1612) ubican estos métodos a nivel locativo y Jucker los compila en 3 ejes principales:

El método de silla (armchair method). Tiene relación con el método intuitivo donde el investigador imagina ejemplos del fenómeno lingüístico. Este método se caracteriza por no tomar usos reales del lenguaje.

El método de campo (the field method). Tiene relación con el método de observación y tiene como fin recolectar elementos reales de uso del lenguaje a través de textos orales o escritos y se consignan en el diario de campo ${ }^{8}$.

El método de laboratorio (the laboratory method): tiene relación con el método de experimentación que se distingue por hacer simulaciones de un evento lingüístico específico con la ayuda de informantes. Normalmente, el investigador le pide a los participantes que imagen una situación comunicativa determinada y cómo se desenvolverían ante ésta. Las dramatizaciones y los cuestionarios de completación de discurso (DCTs) han sido utilizados en este método. Blum-Kulka et al. (1989) han utilizado los DCTs de una manera sistemática y satisfactoria en el campo de la pragmática.

Este articulo basa su análisis de los cumplidos siguiendo los métodos de campo y laboratorio y tomando muestras de los cumplidos a través

6 La palabra bizcocho tiene significado de persona bonita y puede funcionar también como un vocativo.

7 Mera puede tener el significado de "muy buena".

8 Manes y Wolfson (1981) utilizan la terminología enfoque etnográfico. 
de un cuestionario de completación (DCT) y un diario de campo. Un total de 150 cumplidos fueron tomados como muestra de las diferentes fuentes de información.

\section{Fuentes de recolección de información}

\section{Cuestionario de completación (DCT)}

En su tesis de maestría, Calderón (2009) aplicó un cuestionario escrito a 120 antioqueños de estratos y sexos diferentes sobre varias situaciones hipotéticas de cortesía entre las que se encontraban 3 escenarios relacionados con los cumplidos.

- Su papá se ha quitado el bigote. A usted le parece que así se ve muy bien y quiere decírselo. Escriba lo que usted le diría, tal como se lo diría exactamente:

- Un amigo suyo ha bajado de peso. A usted le parece que ha mejorado notablemente su aspecto y quiere decírselo. Escriba lo que usted le diría, tal como se lo diría exactamente.

- Usted está en la sala de espera del consultorio médico y una desconocida que está en la misma sala está cargando un bebé que a usted le parece encantador y quiere decírselo. Escriba lo que usted le diría, tal como se lo diría exactamente.

Diario de campo

En este instrumento, se registraron 30 fenómenos lingüísticos relacionados con los cumplidos utilizados por los habitantes del área metropolitana del Valle del Aburrá en la ciudad de Medellín, Colombia. El investigador tomaba nota inmediata cada vez que se producía un cumplido y registraba también la información etnográfica de los interlocutores: sexo, edad, lugar y nivel de estudio.

\section{Preguntas guía de investigación}

Las preguntas que dirigen el análisis de los cumplidos son:

- ¿Qué tipos de cumplidos se utilizan en Medellín y su área metropolitana del Valle de Aburrá?

9 Los municipios que abarca el Valle de Aburrá en Medellín, Colombia, son: Caldas, la Estrella, Sabaneta, Envigado, Itagüí, Bello, Barbosa, Girardota y Copacabana.

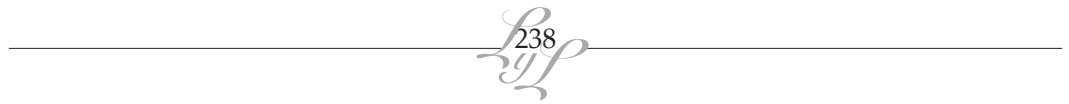


- ¿Qué elementos lingüísticos sobresalen en los cumplidos realizados por los interlocutores de Medellín y su área metropolitana del Valle de Aburrá?

\section{Resultados}

150 registros de cumplidos fueron analizados y categorizados teniendo en cuenta su objeto y forma de realización; a continuación, se brindan algunas muestras con su respectivo análisis:

\section{Objeto del cumplido}

Tabla 1. Ejemplos de cumplidos de apariencia

\begin{tabular}{|c|c|c|c|c|}
\hline Fuente & Cumplido & $\begin{array}{l}\text { Quién lo hace } \\
\text { y destinatario }\end{array}$ & $\begin{array}{l}\text { Forma de } \\
\text { realización }\end{array}$ & $\begin{array}{l}\text { Análisis - } \\
\text { comentario }\end{array}$ \\
\hline $\begin{array}{l}\text { Cuestionario de } \\
\text { completación } \\
(\mathrm{DCT})\end{array}$ & $\begin{array}{l}\text { ¡Ay!!! ¡Cómo } \\
\text { quedó de lindo! }\end{array}$ & $\begin{array}{l}\text { Mujer a } \\
\text { hombre (de } \\
\text { hija a padre) }\end{array}$ & Explícita & $\begin{array}{l}\text { Se constata el uso } \\
\text { frecuente de la } \\
\text { interjección ay }\end{array}$ \\
\hline $\begin{array}{l}\text { Cuestionario de } \\
\text { completación } \\
\text { (DCT) }\end{array}$ & $\begin{array}{l}\text { hey pá, como te } \\
\text { ves de bien sin } \\
\text { bigote. }\end{array}$ & $\begin{array}{l}\text { Hombre a } \\
\text { hombre (de } \\
\text { hijo a padre) }\end{array}$ & Explícita & $\begin{array}{l}\text { Los verbos ver } \\
\text { y quedar son los } \\
\text { que sobresalen } \\
\text { para brindar el } \\
\text { cumplido de } \\
\text { apariencia. }\end{array}$ \\
\hline $\begin{array}{l}\text { Cuestionario de } \\
\text { completación } \\
\text { (DCT) }\end{array}$ & $\begin{array}{l}\text { Oye, te ves } \\
\text { mucho mejor asi } \\
\text { delgado }\end{array}$ & $\begin{array}{l}\text { Mujer a } \\
\text { hombre } \\
\text { (amigos) }\end{array}$ & Explícita & $\begin{array}{l}\text { Uso de la palabra } \\
\text { oye para llamar } \\
\text { la atención del } \\
\text { interlocutor }\end{array}$ \\
\hline $\begin{array}{l}\text { Diario de } \\
\text { campo }\end{array}$ & $\begin{array}{l}\text { ¡Uh, qué camisa } \\
\text { tan bonita, mero } \\
\text { papi! }\end{array}$ & $\begin{array}{l}\text { Mujer a } \\
\text { hombre } \\
\text { (compañeros } \\
\text { de trabajo) }\end{array}$ & Explícita & $\begin{array}{l}\text { Uso de la } \\
\text { interjección } \\
\text { uh como } \\
\text { acompañante } \\
\text { introductoria } \\
\text { del cumplido. } \\
\text { El cumplido se } \\
\text { utiliza como una } \\
\text { forma de saludo. }\end{array}$ \\
\hline $\begin{array}{l}\text { Diario de } \\
\text { campo }\end{array}$ & $\begin{array}{l}\text { Muy bonita la } \\
\text { camisa profe, } \\
\text { me gustan los } \\
\text { cuadros. } \\
\text { (sin respuesta) }\end{array}$ & $\begin{array}{l}\text { Mujer a } \\
\text { hombre } \\
\text { (vendedora a } \\
\text { profesor) }\end{array}$ & Explícita & $\begin{array}{l}\text { Uso del } \\
\text { cumplido como } \\
\text { macroestrategia } \\
\text { para obtener } \\
\text { un fin (vender } \\
\text { un producto al } \\
\text { profesor). }\end{array}$ \\
\hline $\begin{array}{l}\text { Diario de } \\
\text { campo }\end{array}$ & $\begin{array}{l}\text { ¡Ah, qué pulsera } \\
\text { tan bacana! }\end{array}$ & $\begin{array}{l}\text { Mujer } \\
\text { a mujer } \\
\text { (compañeras } \\
\text { de trabajo) }\end{array}$ & Explícita & $\begin{array}{l}\text { Uso de la } \\
\text { palabra tan para } \\
\text { intensificar el } \\
\text { cumplido. }\end{array}$ \\
\hline
\end{tabular}




\section{Tabla 2. Ejemplos de cumplidos de posesión}

\begin{tabular}{|c|c|c|c|c|}
\hline Fuente & Cumplido & $\begin{array}{l}\text { Quién lo hace } \\
\text { y destinatario }\end{array}$ & $\begin{array}{l}\text { Forma de } \\
\text { realización }\end{array}$ & $\begin{array}{l}\text { Análisis - } \\
\text { comentario }\end{array}$ \\
\hline $\begin{array}{l}\text { Diario de } \\
\text { campo }\end{array}$ & $\begin{array}{l}\text { ¡Hey, mera } \\
\text { moto la que } \\
\text { compró el } \\
\text { parcero de tu } \\
\text { hermano! }\end{array}$ & $\begin{array}{l}\text { Hombre } \\
\text { a hombre } \\
\text { (compañeros } \\
\text { de trabajo) }\end{array}$ & Indirecta & $\begin{array}{l}\text { Uso de la } \\
\text { interjección } \\
\text { "Hey" para } \\
\text { introducir el } \\
\text { cumplido. Uso de } \\
\text { las palabras del } \\
\text { parlache "mera y } \\
\text { parcero" }\end{array}$ \\
\hline $\begin{array}{l}\text { Diario de } \\
\text { campo }\end{array}$ & $\begin{array}{l}\text { ¡Ah, mija } \\
\text { qué bolso } \\
\text { tan bonito! } \\
\text { Respuesta: ah, } \\
\text { ya está viejito, } \\
\text { lo compré } \\
\text { hace dos } \\
\text { años, ya no } \\
\text { lo utilizo. Me } \\
\text { choca mucho } \\
\text { cambiar de } \\
\text { bolso. }\end{array}$ & $\begin{array}{l}\text { Mujer a mujer } \\
\text { (compañeras } \\
\text { de trabajo) }\end{array}$ & Explícita & $\begin{array}{l}\text { Uso de la } \\
\text { interjección "Ah" } \\
\text { para introducir } \\
\text { el cumplido. Uso } \\
\text { del vocativo mija } \\
\text { como elemento } \\
\text { de inclusión del } \\
\text { participante. Uso } \\
\text { del adverbio tan } \\
\text { para intensificar. } \\
\text { En la respuesta } \\
\text { se utiliza el } \\
\text { diminutivo "ito" } \\
\text { para reducir } \\
\text { la fuerza del } \\
\text { cumplido. }\end{array}$ \\
\hline $\begin{array}{l}\text { Cuestionario de } \\
\text { completación } \\
\text { (DCT) }\end{array}$ & $\begin{array}{l}\text { ¿Qué bebé tan } \\
\text { lindo! }\end{array}$ & $\begin{array}{l}\text { De mujer } \\
\text { a mujer } \\
\text { (desconocidos) }\end{array}$ & Explícita & $\begin{array}{l}\text { Sobresale la } \\
\text { expresión "qué } \\
\text { bebé tan ..." con } \\
\text { los adjetivos } \\
\text { hermoso, lindo. } \\
\text { Uso del adverbio } \\
\text { tan para } \\
\text { intensificar. }\end{array}$ \\
\hline $\begin{array}{l}\text { Cuestionario de } \\
\text { completación } \\
\text { (DCT) }\end{array}$ & $\begin{array}{l}\text { ¿Qué bebé tan } \\
\text { hermoso! }\end{array}$ & $\begin{array}{l}\text { De hombre } \\
\text { a mujer } \\
\text { (desconocidos) }\end{array}$ & Explícita & $\begin{array}{l}\text { También } \\
\text { sobresale la } \\
\text { expresión "qué } \\
\text { bebé tan ..." con } \\
\text { los adjetivos } \\
\text { hermoso, lindo. } \\
\text { Uso del adverbio } \\
\text { tan para } \\
\text { intensificar. }\end{array}$ \\
\hline
\end{tabular}


Tabla 3. Ejemplos de cumplidos de habilidad

\begin{tabular}{|c|c|c|c|c|}
\hline Fuente & Cumplido & $\begin{array}{l}\text { Quién lo hace } \\
\text { y destinatario }\end{array}$ & $\begin{array}{l}\text { Forma de } \\
\text { realización }\end{array}$ & $\begin{array}{l}\text { Análisis - } \\
\text { comentario }\end{array}$ \\
\hline $\begin{array}{l}\text { Diario de } \\
\text { campo }\end{array}$ & $\begin{array}{l}\text { Contexto: un } \\
\text { estudiante } \\
\text { universitario } \\
\text { le pide a otro } \\
\text { que le muestre } \\
\text { un trabajo } \\
\text { de diseño. } \\
\text { Cuando le } \\
\text { enseña el } \\
\text { trabajo, la otra } \\
\text { persona hace } \\
\text { el cumplido: } \\
\text { ¡huy, mero } \\
\text { teso! } \\
\text { Sin respuesta }\end{array}$ & $\begin{array}{l}\text { Mujer a } \\
\text { hombre }\end{array}$ & Explícita & $\begin{array}{l}\text { La expresión } \\
\text { del parlache } \\
\text { "mero teso" } \\
\text { puede significar } \\
\text { "tener buena } \\
\text { habilidad". } \\
\text { La palabra } \\
\text { mero se puede } \\
\text { tomar como un } \\
\text { adverbio que } \\
\text { intensifica. Uso } \\
\text { de la interjección } \\
\text { "Huy" para } \\
\text { introducir el } \\
\text { cumplido. }\end{array}$ \\
\hline $\begin{array}{l}\text { Diario de } \\
\text { campo }\end{array}$ & $\begin{array}{l}\text { Contexto: } \\
\text { algunos } \\
\text { jugadores de } \\
\text { fútbol están en } \\
\text { un camerino } \\
\text { comentando } \\
\text { acerca del } \\
\text { partido que } \\
\text { acabaron de } \\
\text { terminar: Huy } \\
\text { parce, vos sos } \\
\text { mero monstruo } \\
\text { pa' hacer pases } \\
\text { largos. }\end{array}$ & $\begin{array}{l}\text { Hombre a } \\
\text { hombre }\end{array}$ & Explícita & $\begin{array}{l}\text { Uso de la } \\
\text { interjección } \\
\text { "Huy" para } \\
\text { introducir el } \\
\text { cumplido. Uso de } \\
\text { la palabra parce } \\
\text { como vocativo. } \\
\text { Uso del voceo } \\
\text { como elemento } \\
\text { afiliador. Se } \\
\text { hace doble } \\
\text { intensificación: } \\
\text { con el uso de la } \\
\text { palabra mero y } \\
\text { monstruo. }\end{array}$ \\
\hline
\end{tabular}




\section{Análisis general}

Analizando el objeto y forma de realización de los 150 cumplidos, se puede concluir lo siguiente:

\section{Tabla 4. Análisis general de los cumplidos}

\begin{tabular}{|c|c|c|c|}
\hline $\begin{array}{l}\text { Técnica de } \\
\text { recolección de } \\
\text { información }\end{array}$ & $\begin{array}{l}\text { Objeto del } \\
\text { cumplido }\end{array}$ & $\begin{array}{l}\text { Forma de } \\
\text { realización }\end{array}$ & Análisis general \\
\hline $\begin{array}{l}\text { Cuestionario de } \\
\text { completación } \\
\text { (DCT) }\end{array}$ & $\begin{array}{l}\text { Netamente de } \\
\text { apariencia }\end{array}$ & Explícita & $\begin{array}{l}\text { Las expresiones muy lindo } \\
\text { y muy bien y los verbos } \\
\text { ver y quedar son los } \\
\text { elementos que sobresalen } \\
\text { en este cuestionario en los } \\
\text { diferentes sexos y estratos. } \\
\text { Como ejemplos tenemos: } \\
\text { "cómo te ves de bien asi", } \\
\text { "quedas muy bien así } \\
\text { delgado" }\end{array}$ \\
\hline Diario de campo & $\begin{array}{l}\text { Sobresalen también } \\
\text { los de apariencia }\end{array}$ & $\begin{array}{l}\text { Explícita e } \\
\text { indirecta }\end{array}$ & $\begin{array}{l}\text { El uso de los adverbios } \\
\text { muy y tan aumentan la } \\
\text { fuerza de los cumplidos: } \\
\text { tan bonita..., muy } \\
\text { bonita... }\end{array}$ \\
\hline
\end{tabular}

\section{Observaciones generales}

Del corpus analizado, salen las siguientes observaciones:

- La forma de realización general de los cumplidos se hace de una manera explícita.

- Los cumplidos de apariencia son los que más sobresalen; en contraste, no se encontraron cumplidos de personalidad.

- Se encontraron algunas palabras o expresiones del parlache que se acompañan el cumplido (mero, parce, teso).

- Se puede observar que hay un uso continuo de interjecciones (uh, $\mathrm{ah}$, ).

- También se encontró un uso continuo de los adverbios de intensificación muy y tan.

- Muy pocos ejemplos presentan respuestas a los cumplidos. 


\section{Conclusiones}

Al realizar el análisis de las muestras de los cumplidos en el cuestionario de completación (DCT) y en el diario de campo se percibió uso de cumplidos de apariencia, posesión y habilidad. Los cumplidos de apariencia son los más comunes y apoyan los resultados encontrados por otros investigadores (Manes \& Wolfson, 1981; Knapp, Hopper \& Bell 1984; Holmes, 1988; Herbert, 1990; Cordella et al., 1995). En contraste, no se encontraron cumplidos de personalidad y esto también apoya lo que exponen Manes y Wolfson (1981) quienes argumentan que son los más escasos. Finalmente, la forma sobresaliente de realización de los cumplidos es explícita.

Las interjecciones pueden funcionar como elementos pragmáticos y se pueden usar para llamar la atención del interlocutor (Haverkate, 1994). Se puede notar el uso de las interjecciones para llamar la atención en los cumplidos ah, ay, huy, uh. Se concluye que las interjecciones se usan para intensificar la fuerza del cumplido.

También se encontró un uso frecuente de los adverbios de intensificación muy y tan lo que permite concluir que se utilizan para intensificar la fuerza del cumplido. Finalmente, se pudo observar algunas palabras o expresiones del parlache en los cumplidos. Estas observaciones se pueden mostrar como rasgos característicos de identidad de población de la ciudad de Medellín y su área metropolitana en los procesos de comunicación cotidiana.

Finalmente, se podría concluir que los medellinenses utilizan los cumplidos como una forma de afiliación y se caracterizan por tener interjecciones e intensificadores.

\section{Limitaciones}

El análisis de los cumplidos en los dos instrumentos de recolección de información tiene las siguientes limitaciones:

- En la gran mayoría de las muestras no se incluye información paralingüística. Cook (1992) argumenta que el contacto visual, la proxemia, los gestos sonimportantes en la interacción. Es trascendental analizar los cumplidos con sus contextos paralingüísticos. 
- En los dos instrumentos de recolección de información tampoco se incluye información general de los participantes. Es importante saber si el uso de los cumplidos es producido por participantes de un estrato social determinado, si existe una relación de poder entre ellos. La información sociolingüística es de vital importancia en los contextos de investigación lingüística (Silva-Corvalán, 2001).

- Bustos (2007) sostiene que el cumplido es un acto de habla en el cual el hablante brinda una valoración positiva a su interlocutor y el destinatario normalmente responde ante éste, conllevando al par adyacente. Este análisis no incluyó las respuestas a los cumplidos limitándolos sólo al plano de la emisión debido al número reducido de respuestas.

- Finalmente, se necesita un estudio más riguroso para hacer unas conclusiones más generales y contundentes sobre los cumplidos utilizados en Medellín y su área metropolitana del Valle de Aburrá.

\section{Bibliografía}

Austin, J. (1962). Cómo hacer cosas con palabras. Barcelona: Paidós.

Blum-Kulka, S.; House, J. y Kasper, G. (1989). Cross-cultural pragmatics: requests and apologies. Norwood, NJ, Ablex.

Bustos, J. (2007). "Cumplidos". Clac Círculo 31, 3-12. Consultado el 30 octubre de 2010. En <http://www.ucm.es/info/ circulo/no31/bustos.pdf $>$

Calderón, G. (2009). Estrategias de cortesía verbal en la conversación coloquial. Una aproximación pragmalingüística al comportamiento cortés medellinense. Tesis maestría. Colombia: Universidad de Antioquia.

Campo, E. \& Zuluaga J. (2000). “Complimenting: a matter of cultural constraints". Colombian Applied Linguistics Journal 2 , (1), 27-41.

Cook, G. (1992). The discourse of advertising. London: Routledge

Cordella, M.; Large, H.; Pardo, V. (1995). “Complimenting behavior in Australian English and Spanish speech". Multilingua 14 (3), 235-252. 
Coulmas, F., ed. (1980). Conversational routines. The Hague: Mouton.

De los Heros, S. (2001). Discurso, identidad y género en el castellano peruano. Lima: Pontificia Universidad Católica del Perú, Fondo Editorial.

Escandell, V. (1993). Introducción a la Pragmática. Barcelona: Anthropos. Farghal, M. and Haggan, M. (2006). “Compliment behavior in bilingual Kuwaiti college students". International Journal of Bilingual Education and Bilingualism 9 (1), 94-118.

Golato, A. (2002). “German compliment responses". Journal of Pragmatics 34 (5), 547-571.

Haverkate, H. (1994). La cortesía verbal. Madrid: Gredos, S.A.

Henao, J. y Castañeda, L. (2001). El parlache. Medellín: Editorial Universidad de Antioquia.

Herbert, R. (1987). Sex-based differences in compliment behavior. Paper Presented at the Annual Meeting of the American Anthropological Association, Washington, DC.

. (1988). "Paying Compliments: A Sex-Preferential Politeness Strategy". Journal of Pragmatics 12, 445-465.

. (1986). "Say 'thank you' -or something". American Speech 61, 76-88.

. (1989). "The ethnography of English compliments and compliment response: A contrastive sketch". In: W. Oleksy (ed.), Contrastive pragmatics 3-35. Philadelphia, PA: Benjamins.

. (1997). "The sociology of compliment work in Polish and English". En: Couplan, N. y Jaworski, A. (eds.). Sociolinguistics: a reader and coursebook. Londres: Macmillan, 487-500.

Herbert, R. K. (1990). "Sex-based differences in compliment behavior". Language in Society 19, 201-224. Consultado el 8 de septiembre de 2010 en <http://www.carla.umn. edu/speechacts / compliments / american.html> 
Holmes, J. (1986). “Compliments and compliment responses in New Zealand English". Anthropological Linguistics 28(4): 485-508.

. and Brown, D.(1987). "Teachers and students learning about compliments". TESOL Quarterly, 523-546.

Homenaje a Haverkate (2010). V Coloquio Internacional del programa Edice. Grupo de Estudios Lingüísticos Regionales. Colombia: Universidad de Antioquia.

Jucker, Andreas (2008). "Speech act research between armchair, field and laboratory: The case of compliments". Journal of Pragmatics 41, 1611-1635.

Knapp, M. L. et al. (1984). "Compliments: A Descriptive Taxonomy". Journal of Communication 34 (4), 12-31. Consultado el 20 de septiembre de 2010 en <http:/ / www.carla. umn.edu / speechacts / compliments / american. html>

Lorenzo-Dus, N. (2001). "Compliments responses among British and Spanish university students: a contrastive study". Journal of Pragmatics 33, 107-127.

Manes, J. (1983). “Compliments: Amirror of cultural values”. In N. Wolfson and E. Judd (Eds.). Sociolinguistics and Language Acquisition (82-95). Rowley, MA: NewburyHouse. Consultado el 5 de noviembre de 2010. Recuperado el 13 de septiembre de 2010 en <http: / www.carla. umn.edu / speechacts / compliments / american. html>

Manes, J. \& Wolfson, N. (1981). "The compliment formula". In F. Coulmas (Ed.), Conversational Routine: Explorations in Standardized Communication Situations and Prepatterned Speech (116-132). The Hague, the Netherlands: Mouton Publishers. Consultado el 5 de noviembre de 2010 en <http:/ / www.carla.umn. edu/speechacts / compliments / american.html>

Murillo, J. (2003). “Significados de la cortesía lingüística a partir de la aplicación de un test de hábitos sociales en

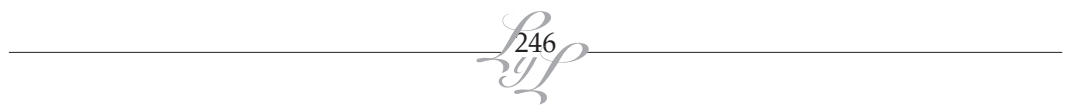


Costa Rica". En Bravo, D. (Ed.). La perspectiva no etnocentrista de la cortesía: identidad sociocultural de las comunidades hispanohablantes, ACTAS del Primer Coloquio del Programa de EDICE, Estocolmo.

Mustapha, A. (2004). Gender variation in Nigerian English compliments. Ph.D. dissertation, University of Essex.

Norrick, N. (1980). “The speech act of complimenting". In: E. Hovdhaugen (Ed.). The Nordic language and modern linguistics, 296-304. Oslo: Universitetsforlaget.

Rees-Miller, J. (2011). “Compliments revisited: Comtemporary compliments and gender". Journal of Pragmatics 43, 2673-2688.

Silva-Corvalán, C. (2001). Sociolingüística y pragmática del español. Washington: Georgetown University Press.

Searle, J. (1969). Speech acts: an essay in the philosophy of language. Cambridge: Cambridge University Press.

Tang, Chen-Hsin, and Zhang, Grace Qiao. (2009). “A contrastive study of compliment responses among Australian English and Mandarin Chinese speakers". Journal of Pragmatics 41 (2), 325-345.

Wolfson, N. (1983). "An Empirically based analysis of complimenting in American English". In N. Wolfson and E. Judd (Eds.). Sociolinguistics and Language Acquisition, 8295. Rowley, MA: Newbury House. Consultado el 10 de diciembre de 2010 en <http: / / www.carla.umn. edu/speechacts / compliments / american.html>

Wolfson, N. (1981). "Compliments in cross-cultural perspective”. TESOL Quarterly, 15, 2: 117-24. 